\title{
Thermal fluctuations and electromagnetic noise spectra in quantum statistical mechanics.
}

\author{
Ladislaus Alexander Bányai*, Mircea Bundaru** \\ and Paul Gartner***
}

Institut für Theoretische Physik, Goethe-Universität, Frankfurt am Main* National Institute of Physics and Nuclear Engineering - Horia Hulubei, Bucharest**

National Institute of Materials Physics, PO Box MG-7, Bucharest-Magurele, Romania***

banyai@itp.uni-frankfurt.de, corresponding author

\begin{abstract}
We derive the thermal noise spectrum of the Fourier transform of the electric field operator of a given wave vector starting from the quantumstatistical definitions and relate it to the complex frequency and wave vector dependent complex conductivity in a homogeneous, isotropic system of electromagnetic interacting electrons. We analyze separately the longitudinal and transverse case with their peculiarities. The Nyquist formula for vanishing frequency and wave vector, as well as its modification for non-vanishing frequencies and wave vectors follow immediately. Furthermore we discuss also the noise of the photon occupation numbers. It is important to stress that no additional assumptions at all were used in this straightforward proof.
\end{abstract}

keywords: fluctuations, noise spectra, longitudinal and transverse electric fields, Nyquist noise, photon number noise

\section{Introduction}

There are in the literature several theoretical approaches to the thermal noise in solids. The basic result is Nyquist's law [1], [2] for voltage noise at zero frequency. Later Callen and Welton [3] established a formula relating the noise spectrum to the frequency dependent resistance based on a fluctuationdissipation theorem. In an earlier paper [4] a derivation based directly on the spectrum of the voltage fluctuations was given. The resulting formula was not identical with that of Ref. [3] replacing the frequency dependent resistance by a frequency-dependent impedance. In the mean-time several derivations under 
different stochastic, thermodynamic electric circuitry approaches with differing even conflicting results were published (see Refs.[5],[6],[7]). In a more recent book of one of the authors [8] an alternative derivation of the result of [4] was given, starting from the electric field fluctuations. See also the recent review articles [9], [10], that however overlook a delicate point of electrodynamics in the frame of the linear response theory and Ref. [10] uses a definition of a "quantum fluctuation" we do not share. We do not intend here to comment on recent discussion [11] about the origin of discrepancies to the measured noise spectra.

In this paper we want to describe our theoretical approach in a systematical way since we believe this is the only one staying on the sound basis of quantum statistical mechanics and electrodynamics. It is obtained without any supplementary assumptions. One starts from the quantum-mechanical definition [12] of the fluctuation of an observable in a system in macro-canonical equilibrium defined as the average quadratic deviation in time. The noise spectrum is just its Fourier transform. We use the definition of the complex frequency and wave-vector dependent dielectric function (or conductivity) by the well-known modification of Kubo's linear response theory [13] in an e.m. interacting system due to [14] and [15]. The only implicit thermodynamic element here is the expectation, that linear response theory is correct i.e. the thermodynamic limit introduces irreversibility. Additionally, our approach stresses the role of internal fields in the linear response of interacting systems. We show that this leads to differences with respect to previous results in the literature.

Along this line we derive the thermal noise spectrum of a longitudinal electric field of wave-vector $\vec{k}$ in a homogeneous isotropic system. We obtain a generalized Nyquist formula relating it for non-vanishing frequency $\omega$ (of the noise) and wave-vector $\vec{k}$ (of the field) to the frequency and wave-vector dependent longitudinal dielectric function (or conductivity).

We give also a comparison of this derivation to the alternative proof of the frequency dependent Nyquist formula given in Ref. [4] underlining the essential role of the charge density fluctuations in the longitudinal case. In this context we discuss the deficiency of the Callen-Welton fluctuation-dissipation theorem [3] which was formulated years before the realization of the role of the internal field produced by the charged particles.

Thereafter, we derive the never touched noise spectrum of the transverse electric field (radiation field) starting from the non-relativistic QED by neglecting the retardation (i.e. in the $1 / c^{2}$ approximation). A Nyquist -like relation occurs again. Though, it has certain peculiarities due to the fact that unlike by the longitudinal field, the noise may be related to the conductivity only when the frequency $\omega$ of the noise is related to the wave vector of the field as $\omega=c k$.

Within the same $1 / c^{2}$ approximation of the QED we discuss also the noise spectrum of the photon occupation numbers. This also may be expressed through the electronic transverse current-current correlator. However due to the photonic factors under the integrals it cannot be related to the transverse conductivity. 


\section{Fluctuations and their spectral density.}

The time fluctuation $\Delta_{X}(t)$ of a given observable (hermitian operator) $X$ in thermal equilibrium is defined [12] as the average square deviation

$$
\Delta_{X}(t)=\left\langle(X(t)-X(0))^{2}\right\rangle_{0} \geq 0,
$$

where the average is taken over the macro-canonical equilibrium density matrix

$$
\rho_{0} \equiv \frac{e^{-\beta(H-\mu N)}}{Z}
$$

and the time evolution of the operator $X$ is given by

$$
X(t)=e^{\frac{\imath H t}{\hbar}} X e^{\frac{-\imath H(t)}{\hbar}} .
$$

This is analogous to the general definition of a fluctuation both in classical- or quantum statistics.

Expanding Eq. 1 one has

$$
\begin{aligned}
\Delta_{X}(t) & =\left\langle X(t)^{2}+X(0)^{2}-X(t) X(0)-X(0) X(t)\right\rangle_{0} \\
& =2\left\langle X(0)^{2}\right\rangle-\langle X(t) X(0)+X(0) X(t)\rangle_{0}
\end{aligned}
$$

Leaving apart the constant $2\left\langle X(0)^{2}\right\rangle$ usually chosen to be vanishing, the entity of interest is

$$
\delta_{X}(t)=\langle X(t) X(0)+X(0) X(t)\rangle_{0}
$$

which is a real and even function of $t$. As a consequence its Fourier transform

$$
\tilde{\delta}_{X}(\omega)=\int_{-\infty}^{\infty} d t e^{\imath \omega t} \delta_{X}(t)=2 \int_{0}^{\infty} d t \cos (\omega t) \delta_{X}(t)
$$

is also real and even. Moreover, according to the Wiener-Khinchin Theorem $[16,17],[18]$ it is positive. This is defined [12] as the "noise" spectrum of $X$ in quantum statistics ${ }^{1}$.

It is easy to show, by expansion in the basis of the eigenfunctions of $H-\mu N$ for any observable $X$ (here $X \equiv X(0)$ ) the following two identities

$$
\begin{aligned}
\tilde{\delta}_{X}(\omega) & =2 \operatorname{coth}\left(\frac{\beta \hbar \omega}{2}\right) \Re\left\{\int_{0}^{\infty} d t e^{-\imath \omega t}\langle[X, X(t)]\rangle_{0}\right\} \\
& =2 \hbar \omega \operatorname{coth}\left(\frac{\beta \hbar \omega}{2}\right) \Re \int_{0}^{\infty} d t e^{-\imath \omega t} \int_{0}^{\beta} d \lambda\left\langle X X(t+\imath \hbar \lambda\rangle_{0} .\right.
\end{aligned}
$$

Correlators of both types appear in the linear response theory. Our purpose is to relate the electromagnetic thermal noise to the coefficients of that formalism.

\footnotetext{
${ }^{1}$ An asymmetrical "quantum noise" $\int_{-\infty}^{\infty} d t e^{\imath \omega t}\langle X(t) X\rangle_{0}$ as in Ref.[10] has no direct measurable physical meaning since $\langle X(t) X\rangle_{0}$ is not the average of a hermitian operator.
} 


\section{Linear response theory.}

\subsection{Linear response to an applied electromagnetic field.}

In a system of electromagnetic interacting charged particles the perturbing Hamiltonian contains only the applied (external) fields. However, the charged particles themselves are sources of electromagnetic fields. This makes the formulation of the problem more delicate. The linear part of the perturbation due to applied electromagnetic scalar $V^{e x t}(\vec{x}, t)$ and vector potentials $\vec{A}^{e x t}(\vec{x}, t)$ is

$$
H^{\prime}(t)=\int d \vec{x}\left\{\rho(\vec{x}) V^{e x t}(\vec{x}, t)-\vec{j}(\vec{x}) \vec{A}^{e x t}(\vec{x}, t)\right\} .
$$

The average internal e.m. field has yet to be calculated. Together with the external field it gives rise to the total e.m. field in the system (matter). The conductivity is defined through the relationship between the average current and this total field. This relationship may be given explicitly only in homogeneous, isotropic systems after Fourier transformation in time and space variables.

The average of the current density operator

$$
\vec{j}(\vec{x}, t)=\frac{e}{2 m} \psi(\vec{x})^{*}\left(-\imath \hbar \nabla+\frac{e}{c} \vec{A}^{e x t}(\vec{x}, t)\right) \psi(\vec{x})+h . c
$$

given by the generalization ${ }^{2}$ of Kubo's adiabatic linear response theory [13] to the above general electromagnetic perturbation is

$$
\left\langle j_{\mu}(\vec{x}, t)\right\rangle=\lim _{s \rightarrow+0} \int_{-\infty}^{t} d t^{\prime} e^{s t^{\prime}} \int_{0}^{\beta} d \lambda \int d \vec{x}^{\prime}\left\langle j_{\nu}\left(\vec{x}^{\prime},-\imath \hbar \lambda\right) j_{\mu}\left(\vec{x}, t-t^{\prime}\right)\right\rangle_{0} E_{\nu}^{e x t}\left(\vec{x}^{\prime}, t^{\prime}\right)
$$

where $\mu, \nu=1,2,3$ are vector-indices and summation over double indices is understood, while $s$ is the adiabatic parameter we shall omit for simplicity in the following. (The current density operators in the correlator being already those in the absence of the external field!)

This relationship is gauge invariant and valid for any external electric field stemming from the e.m. potentials

$$
\vec{E}^{e x t}=-\nabla V^{e x t}-\frac{1}{c} \frac{\partial}{\partial t} \vec{A}^{e x t}
$$

It may be obtained by linear response to the perturbation Eq.9, which involves its time-derivative, followed by the use of the continuity equation for $\partial \rho / \partial t$ and partial integration assuming the vanishing of the correlator at infinite spaceand time- distances.

If the e.m. interaction between the charged particles is ignored, one may reinterpret the e.m. field $\vec{E}^{\text {ext }}$ as the mean field $\vec{E}^{\text {mean }}$. Only within this approximation the kernel of Eq. 11 may be related directly to the conductivity of that system.

\footnotetext{
${ }^{2}$ In his original paper Kubo considered only the perturbation by a constant homogeneous electric field.
} 
As it stays Eq.11 actually needs the formulation of the whole problem within the non-relativistic QED (see for example Ref. [8] for the definition of its Hamiltonian $H_{Q E D}$ ), not just the usual Coulomb Hamiltonian used in condensed matter theory. This emerges from the necessity to consider the averages of both the longitudinal and transverse electric fields.

However, after the first few steps we shall resort to a simplified approach by neglecting retardation i.e. in an $1 / c^{2}$ approximation of the QED in which the motion of the electrons and photons are separated. The photons are considered to be free, while the charged particles interact directly by charge-charge and current-current forces. Their e.m. Hamiltonian [19], [20] (here for sake of simplicity just for electrons) is

$$
\begin{aligned}
H_{e . m .} & =\int d \vec{x} \psi^{+}(\vec{x})\left[\frac{\hbar^{2}}{2 m}\left(\nabla-\frac{\imath e}{\hbar c} \vec{A}^{e x t}(\vec{x}, t)\right)^{2}+e V^{e x t}(\vec{x}, t)\right] \psi(\vec{x}) \\
& +\frac{1}{2} \int d \vec{x} \int d \vec{x}^{\prime} \frac{N\left[\rho(\vec{x}) \rho\left(\vec{x}^{\prime}\right)-\frac{1}{c^{2}} \vec{j}_{T}(\vec{x}) \vec{j}_{T}\left(\vec{x}^{\prime}\right)\right]}{\left|\vec{x}-\vec{x}^{\prime}\right|}
\end{aligned}
$$

where $\vec{j}_{T}(\vec{x})$ is the transverse current density

$$
\vec{j}_{T}(\vec{r}, t) \equiv \vec{j}(\vec{r}, t)+\frac{1}{4 \pi} \nabla \int d \vec{r}^{\prime} \frac{\nabla^{\prime} \vec{j}\left(\vec{r}^{\prime}, t\right)}{\left|\vec{r}-\vec{r}^{\prime}\right|}
$$

and the symbol $N[\ldots]$ means taking the normal product of the operators.

Of course beside this electromagnetic part one may include any other interaction of the electrons like those with the crystal lattice, phonons, impurities, etc.

\subsection{Frequency and wave vector dependent conductivity in a homogeneous, isotropic system.}

In a homogeneous (translation invariant) system one obtains by Fourier transformation in time and coordinate of Eq. 11

$$
\left\langle\tilde{j}_{\mu}(\vec{k}, \omega)\right\rangle=\int_{0}^{\infty} d t \int_{0}^{\beta} d \lambda \int d \vec{x} e^{\imath(\vec{k} \vec{x}+\omega t)}\left\langle j_{\nu}(0,0) j_{\mu}(\vec{x}, t+\imath \hbar \lambda)\right\rangle_{0} E_{\nu}^{e x t}(\omega, \vec{k})
$$

i.e. one has a local relationship in the Fourier space

$$
\left\langle\tilde{j}_{\mu}(\vec{k}, \omega)\right\rangle=\kappa(\vec{k}, \omega)_{\mu \nu} E_{\nu}^{e x t}(\omega, \vec{k})
$$

with

$$
\kappa(\vec{k}, \omega)_{\mu \nu}=\int_{0}^{\infty} d t \int_{0}^{\beta} d \lambda \int d \vec{x} e^{\imath(\vec{k} \vec{x}+\omega t)}\left\langle j_{\nu}(0,0) j_{\mu}(\vec{x}, t+\imath \hbar \lambda)\right\rangle_{0}
$$

If the system under consideration is isotropic, then one may separate the longitudinal and transverse parts 


$$
\kappa(\vec{k}, \omega)_{\mu \nu}=\frac{k_{\mu} k_{\nu}}{k^{2}} \kappa_{L}(k, \omega)+\left(\delta_{\mu \nu}-\frac{k_{\mu} k_{\nu}}{k^{2}}\right) \kappa_{T}(k, \omega)
$$

and

$$
\begin{aligned}
\left\langle\overrightarrow{\tilde{j}}_{L}(\vec{k}, \omega)\right\rangle & =\kappa_{L}(k, \omega) \overrightarrow{\tilde{E}}_{L}^{e x t}(\vec{k}, \omega) \\
\left\langle\overrightarrow{\tilde{j}}_{T}(\vec{k}, \omega)\right\rangle & =\kappa_{T}(k, \omega) \overrightarrow{\tilde{E}}_{T}^{\text {ext }}(\vec{k}, \omega) .
\end{aligned}
$$

It is important to remark here that the frequency and the wave vector in the transverse case are not independent $(\omega=c k)$.

One may define instead of the complex conductivity a complex dielectric function related to the complex conductivity by

$$
\epsilon(k, \omega)=1+\frac{4 \pi}{\imath \omega} \sigma(k, \omega)
$$

\subsubsection{The longitudinal dielectric function.}

In the longitudinal case it is convenient to consider the dielectric function $\epsilon(\vec{k}, \omega)$ Eq. 21 using the relationship between the charge density and the scalar potential in the Coulomb gauge

$$
\langle\tilde{\rho}(\vec{k}, \omega)\rangle=\tilde{K}_{L}(k, \omega) \tilde{V}^{e x t}(\vec{k}, \omega)
$$

with

$$
\tilde{K}_{L}(\vec{k}, \omega)=\int_{0}^{\infty} d t \int d \vec{x} e^{i \vec{k} \vec{x}} e^{\imath \omega t} K_{L}(\vec{x}, t)
$$

and

$$
K_{L}(\vec{x}, t) \equiv \frac{\theta(t)}{\imath \hbar}\langle[\rho(\vec{x}, t), \rho(0,0)]\rangle_{0}
$$

obtained directly from the linear response to the perturbation with the Hamiltonian of Eq. 9 in the presence of just a scalar external potential.

Since in the Coulomb gauge the average internal and the external scalar potentials are given by the Poisson equations

$$
\begin{array}{r}
k^{2}\left\langle\tilde{V}^{i n t}(\vec{k}, \omega)\right\rangle=4 \pi\langle\tilde{\rho}(\vec{k}, \omega)\rangle \\
k^{2} \tilde{V}^{e x t}(\vec{k}, \omega)=4 \pi \tilde{\rho}^{e x t}(\vec{k}, \omega)
\end{array}
$$

and the longitudinal dielectric function is defined by the relationship between the external charge density and the total potential $V=V^{i n t}+V^{\text {ext }}$

$$
k^{2} \epsilon_{L}(\vec{k}, \omega) \tilde{V}(\vec{k}, \omega)=4 \pi \tilde{\rho}^{e x t}(\vec{k}, \omega)
$$

by using Eq. 22 one gets

$$
\epsilon_{L}(\vec{k}, \omega) \equiv \frac{1}{1+\frac{4 \pi}{k^{2}} \tilde{K}_{L}(\vec{k}, \omega)} .
$$




\subsubsection{The transverse conductivity.}

From

$$
\left\langle\overrightarrow{\tilde{j}}_{T}(\vec{k}, \omega)\right\rangle=\kappa_{T}(k, \omega) \overrightarrow{\tilde{E}}_{T}^{e x t}(\vec{k}, \omega)
$$

neglecting the retardation

$$
\left\langle\overrightarrow{\tilde{E}}_{T}^{i n t}(\vec{k}, \omega\rangle=\imath \omega \frac{4 \pi}{c^{2} k^{2}}\left\langle\overrightarrow{\tilde{j}}_{T}(\vec{k}, \omega)\right\rangle=\imath \omega \frac{4 \pi}{c^{2} k^{2}} \kappa_{T}(k, \omega) \overrightarrow{\tilde{E}}_{T}^{\text {ext }}(\vec{k}, \omega)\right.
$$

and therefore with the definition

$$
\left\langle\overrightarrow{\tilde{j}}_{T}(\vec{k}, \omega)\right\rangle=\sigma_{T}(k, \omega)\left(\overrightarrow{\tilde{E}}_{T}^{\text {ext }}(\vec{k}, \omega)+\overrightarrow{\tilde{E}}_{T}^{i n t}(\vec{k}, \omega)\right)
$$

we get

$$
\sigma_{T}(k, \omega)=\frac{\kappa_{T}(k, \omega)}{1+\imath \omega \frac{4 \pi}{c^{2} k^{2}} \kappa_{T}(k, \omega)}
$$

Since the transverse external electric field obeys the wave equation, in these equations the variables $\omega$ and $k$ are related by $\omega=c k$ ! Therefore one might speak about a frequency dependent transverse conductivity

$$
\sigma_{T}(\omega)=\frac{\kappa_{T}(\omega / c, \omega)}{1+\imath \frac{4 \pi}{\omega} \kappa_{T}(\omega / c, \omega)}
$$

\subsection{The noise spectrum of the longitudinal electric field.}

From the hermiticity of the operator $\vec{E}(\vec{x}, t)$ it follows that after Fourier transforming in the space variable $\overrightarrow{\tilde{E}}(-\vec{k}, t)^{+}=\overrightarrow{\tilde{E}}(\vec{k}, t)$ and we may define two hermitian scalar operators (observables) as the "real" and "imaginary" parts of the longitudinal field operator $\frac{\vec{k}}{k} \overrightarrow{\tilde{E}}(\vec{k}, t)$

$$
\begin{aligned}
& E_{L}^{R}(\vec{k}, t)=\frac{\vec{k}}{2 k}(\overrightarrow{\tilde{E}}(\vec{k}, t)+\overrightarrow{\tilde{E}}(-\vec{k}, t)) \\
& E_{L}^{I}(\vec{k}, t)=-\imath \frac{\vec{k}}{2 k}(\overrightarrow{\tilde{E}}(\vec{k}, t)-\overrightarrow{\tilde{E}}(-\vec{k}, t))
\end{aligned}
$$

The noise of any of these observables is (omitting the upper indices R,I) according Eq. 7 is

$$
\tilde{\delta}_{E_{L}}(\omega, k)=2 \operatorname{coth}\left(\frac{\beta \hbar \omega}{2}\right) \Re\left\{\int_{0}^{\infty} d t e^{-\imath \omega t}\left\langle\left[E_{L}(\vec{k}, 0), E_{L}(\vec{k}, t)\right]\right\rangle_{0}\right\}
$$

Here the inverted order of arguments $(\omega, k)$ in the notation underlines that $\vec{k}$ is the wave-vector of the electric field, while $\omega$ is the frequency of the noise spectrum. 
The Fourier transform of the longitudinal electric field is related by the Poisson equation $(\nabla \vec{E}(\vec{x}, t)=4 \pi \rho(\vec{x}, t))$ to the Fourier transform of the charge density by

$$
\tilde{E}(\vec{k}, t)=\imath \frac{4 \pi}{k} \tilde{\rho}(\vec{k}, t)
$$

It is obvious that the quantities of interest are $\langle[\tilde{\rho}(\vec{k}), \tilde{\rho}(\vec{q}, t)]\rangle$ with $\vec{q}= \pm \vec{k}$. One has, using translation invariance in homogeneous systems

$$
\begin{aligned}
\langle[\tilde{\rho}(\vec{k}), \tilde{\rho}(\vec{q}, t)]\rangle_{0} & =\int d \vec{x}^{\prime} e^{\imath \vec{q} \vec{x}^{\prime}} \int d \vec{x} e^{\imath \vec{k} \vec{x}}\left\langle\left[\rho\left(\vec{x}-\vec{x}^{\prime}\right), \rho(0, t)\right]\right\rangle_{0} \\
& \left.\left.=\int d \vec{x}^{\prime} e^{\imath(\vec{k}+\vec{q}) \vec{x}^{\prime}} \int d \vec{x} e^{i \vec{k} \vec{x}},\right\rangle[\rho(\vec{x}), \rho(0, t)]\right\rangle_{0} \\
& =\Omega \delta_{\vec{k}+\vec{q}, 0} \int d \vec{x} e^{\vec{k} \vec{x}}\langle[\rho(\vec{x}), \rho(0, t)]\rangle_{0}
\end{aligned}
$$

with $\Omega$ being the volume (under cyclic boundary conditions).

In the case $\vec{q}=\vec{k}$ the $\delta$-condition implies $\vec{k}=0$, and one is left with the commutator of the total charge with the charge density at the origin. The former is a conserved quantity and therefore it can be taken at any time, in particular at the same time $t$ as the local charge, whith the result that their commutator is zero. Therefore only the case $\vec{q}=-\vec{k}$ contributes, and one gets

$$
\begin{aligned}
& \tilde{\delta}_{E_{L}}(\omega, k)= \\
& -\Omega \operatorname{coth}\left(\frac{\beta \hbar \omega}{2}\right) \frac{(4 \pi)^{2}}{k^{2}} \Re\left\{\int_{0}^{\infty} d t e^{-\imath \omega t} \int d \vec{x} e^{-\imath \vec{k} \vec{x}}\langle[\rho(\vec{x}), \rho(0, t)]\rangle_{0}\right\} .
\end{aligned}
$$

On the other hand, for Coulomb systems in the frame of the linear response theory (see Eqs. 23, 24 and 27 ) the following relationship for the longitudinal dielectric function holds

$$
\frac{4 \pi}{\hbar k^{2}} \Re\left\{\int_{0}^{\infty} d t e^{-\imath \omega t} \int d \vec{x} e^{-\imath \vec{k} \vec{x}}\langle[\rho(\vec{x}), \rho(0, t)]\rangle_{0}\right\}=\Im\left\{\frac{1}{\epsilon_{L}(\vec{k}, \omega)}-1\right\}
$$

and we identify

$$
\tilde{\delta}_{E_{L}}(\omega, k)=-4 \pi \hbar \Omega \operatorname{coth}\left(\frac{\beta \hbar \omega}{2}\right) \Im\left\{\frac{1}{\epsilon_{L}(\vec{k}, \omega)}-1\right\}
$$

This is our most general new result for the noise spectrum of the longitudinal electric field of wave vector $\vec{k}$.

For $\vec{k} \rightarrow 0$

$$
\begin{aligned}
\tilde{\delta}_{E_{L}}(\omega, 0) & =-4 \pi \hbar \Omega \operatorname{coth}\left(\frac{\beta \hbar \omega}{2}\right) \Im\left\{\frac{1}{\epsilon_{L}(0, \omega)}\right\} \\
& =4 \pi \Omega \hbar \operatorname{coth}\left(\frac{\beta \hbar \omega}{2}\right) \frac{\Im \epsilon_{L}(0, \omega)}{\Re \epsilon_{L}(0, \omega)^{2}+\Im \epsilon_{L}(0, \omega)^{2}}
\end{aligned}
$$


Since at $\omega \rightarrow 0$ only the imaginary part of the dielectric function diverges as $\frac{4 \pi}{\omega} \sigma_{d c}$, we get in this limit

$$
\tilde{\delta}_{E_{L}}(0,0)=4 k_{B} T \frac{\Omega}{\sigma_{d c}}
$$

Taking into account, that in the case of a constant electric field in space $\mathcal{E}$ the Fourier transform is proportional to the volume $\Omega$, we may conclude that its noise is given by

$$
\left\langle\delta \mathcal{E}^{2}\right\rangle_{0}=\frac{4 k_{B} T}{\Omega \sigma_{d c}}
$$

On the other hand introducing the resistance

$$
R=\frac{L}{\sigma_{d c} S}
$$

and the potential drop as

$$
U=\mathcal{E} L
$$

where $S$ and $L$ are the cross-section and the length of the sample from Eq. 47 follows

$$
\left\langle\delta U^{2}\right\rangle_{0}=4 R k_{B} T
$$

and this is finally the old Nyquist theorem [1] .

\section{Our original derivation.}

The result of the previous Section for the spectral density of the thermal noise Eq.41 was earlier derived by Ref.[4], however in a different manner. One considers a sample of length $L$ (along the $z$ axis) and cross-section $S$ and defines the voltage drop along the sample as

$$
U=\frac{1}{S} \int d x d y[V(x, y, L)-V(x, y, 0)]
$$

where $^{3}$

$$
V(\vec{x})=\int d \vec{x} \frac{\rho\left(\vec{x}^{\prime}\right)}{\left|\vec{x}-\vec{x}^{\prime}\right|}
$$

is the potential created by the internal charge density. In the limit $S \rightarrow \infty$ one has

$$
U=\frac{4 \pi}{S} \int d \vec{x} z \rho(\vec{x})
$$

\footnotetext{
${ }^{3}$ In this paper we use the Gauss system of units, while in [4] the SI system was used.
} 
Using the equation of continuity

$$
\frac{\partial \rho(\vec{x}, t)}{\partial t}+\nabla \vec{j}(\vec{x}, t)=0
$$

and the vanishing of the normal component of the current density at the ends of the sample (isolated sample!) one relates the potential drop to the averaged charge flux in the $z$-direction

$$
I \equiv \frac{S}{\Omega} \int d \vec{x} j_{z}(\vec{x})
$$

by

$$
\frac{\partial U(t)}{\partial t}=4 \pi \frac{L}{S} I(t)
$$

and correspondingly the spectral densities of their fluctuations (with the original notations) are related

$$
\delta U_{\omega}^{2}=\left(\frac{4 \pi L}{S \omega}\right)^{2} \delta I_{\omega}^{2} .
$$

On the other hand, according Eq. 1.8 of the original [4] based on linear response theory and noise formula, $\delta I_{\omega}^{2}$ is related to the longitudinal conductivity by

$$
\delta I_{\omega}^{2}=2 \frac{S}{L} \hbar \omega \operatorname{coth}\left(\frac{\beta \hbar \omega}{2}\right) \Re\left\{\frac{\sigma_{L}(\omega)}{1-\frac{\imath 4 \pi}{\omega} \sigma_{L}(\omega)}\right\}
$$

and one gets

$$
\delta U_{\omega}^{2}=2 \hbar 4 \pi \frac{L}{S} \operatorname{coth}\left(\frac{\beta \hbar \omega}{2}\right) \Re \frac{\frac{4 \pi}{\omega} \sigma_{L}(\omega)}{1-\frac{24 \pi}{\omega} \sigma_{L}(\omega)} .
$$

Or taking into account also the relationship between the complex conductivity and the complex dielectric function Eq.21

$$
\delta U_{\omega}^{2}=8 \pi \hbar \frac{L}{S} \operatorname{coth}\left(\frac{\beta \hbar \omega}{2}\right) \Im \frac{1}{1-\frac{\imath 4 \pi}{\omega} \sigma_{L}(\omega)}=-8 \pi \hbar \frac{L}{S} \operatorname{coth}\left(\frac{\beta \hbar \omega}{2}\right) \Im \frac{1}{\epsilon_{L}(\omega)}
$$

that is identical to Eq. 41.

Now, one has the frequency dependent resistance

$$
R(\omega)=\frac{L}{S \Re \sigma_{L}(\omega)}
$$

along the $z$ direction and one may define a capacity between the end crosssections of the sample

$$
C(\omega)=\frac{S}{L 4 \pi} \Re \epsilon_{L}(\omega)=\frac{S}{L 4 \pi}\left(1+\frac{4 \pi}{\omega} \Im \sigma_{L}(\omega)\right) .
$$


If the resistance and the capacitor are parallel linked, then the resulting impedance $Z(\omega)$ is

$$
\frac{1}{Z(\omega)}=\frac{1}{R(\omega)}+\imath \omega C(\omega)
$$

Then in terms of these extensive entities typical for electric circuits the resulting generalization of the Nyquist formula for any non-vanishing frequency looks as ${ }^{4}$

$$
\delta U_{\omega}^{2}=2 \hbar \omega \operatorname{coth}\left(\frac{\beta \hbar \omega}{2}\right) \Re Z(\omega)
$$

A specific aspect of this somewhat lengthy derivation is that the isolation of the sample (no current flow perpendicular to the boundary cross-section) is explicitly included. However, in both derivations the origin of the fluctuation is the fluctuation of the charge density in the system and the results are identical.

Our generalization of the Nyquist formula Eq.60 for non-zero frequencies differs from that obtained by Callen-Welton [3] by the replacement of the resistance $R(\omega)$ of Eq. 57 by the impedance $Z(\omega)$ Eq.59. This discrepancy is however irrelevant in the case of wires, where the capacity $C(\omega)$ of Eq.58 is negligible.

\section{The Callen-Welton formula.}

Callen and Welton [3] long before Kubo [13] treated linear transport due to an applied potential drop $V$ and related it to the currrent-current correlation. Thereafter they derived the integrated noise spectrum:

$$
\begin{aligned}
\left\langle V^{2}\right\rangle & =\frac{2}{\pi} \int_{0}^{\infty} d \omega\left(\frac{1}{2} \hbar \omega+\hbar \omega\left[e^{\beta \hbar \omega}-1\right]^{-1}\right) R(\omega) \\
& \equiv \frac{1}{\pi} \int_{0}^{\infty} d \omega \hbar \omega \operatorname{coth}\left(\frac{\beta \hbar \omega}{2}\right) R(\omega) .
\end{aligned}
$$

However, these fluctuations in the original paper were implicitly still the fluctuations of the applied voltage and did not include the field created by the charged particles themselves. In Ref.[3] no explicit definition of the noise spectral density is given. Nevertheless the integrand is usually interpreted as the noise spectral density. An instant derivation of this spectrum results indeed if one considers the quantum mechanical current fluctuation as the source of the noise, express it by the Kubo formula of the conductivity and implicitly to the frequency-dependent resistance

$$
\left(\delta I_{\omega}^{2}\right)^{K}=2 \hbar \omega \operatorname{coth}\left(\frac{\beta \hbar \omega}{2}\right) \frac{1}{R(\omega)} .
$$

Multiplying with $R(\omega)^{2}$ one gets indeed the voltage noise of Ref. [3]

\footnotetext{
${ }^{4}$ In the original paper unfortunately a misprint occurred in this equation (namely instead of $\Re Z(\omega)$ stand $\left.\Re Z(\omega)^{-1}\right)$ !
} 


$$
\left(\delta V_{\omega}^{2}\right)^{C W}=2 \hbar \omega \operatorname{coth}\left(\frac{\beta \hbar \omega}{2}\right) R(\omega) .
$$

This result differs from our Eq. 60 by the apparition of the resistance $R(\omega)$ instead of the real part of the impedance $Z(\omega)$ of Eq.59.

There are two sources for this discrepancy. First at all, as we mentioned, here one starts from the current fluctuations. Secondly, as it was already stressed, the Kubo formula does not define properly the conductivity in Coulomb interacting systems. Immediately after Kubo other authors [14],[15] pointed out, that the Kubo formula has to be corrected by including the induced field produced by the electrons themselves. It implies also, that not the complex dielectric function satisfies the dispersion relations, but its inverse [21]. This changes completely both the current noise as well as the resulting voltage noise arising from it along the unmodified fluctuation-dissipation theorem and does not lead any more in the $\omega \rightarrow 0$ limit to the Nyquist formula Eq. 47 .

This was our motivation to derive the voltage noise not indirectly from the current fluctuation, but directly from the fluctuations of the voltage, or electric field intensity. This way leads to the Nyquist result at $\omega \rightarrow 0$, while for nonvanishing frequency it is the more general expression Eq.60. However, as we already mentioned, the discrepancy in the macroscopic result may be irrelevant if the capacity $C(\omega)$ of Eq. 58 is negligible.

\section{The noise spectrum of the transverse electric field.}

Let us consider now the noise of the transverse (radiation) electric field $\vec{E}_{T}(\vec{x}, t)$ in a homogeneous, isotropic sample. There are two possible polarizations of this field defined by the two orthogonal to each other and to the wave vector $\vec{k}$ unit vectors $\vec{e}_{\vec{k}}^{(\lambda)}=\vec{e}_{-\vec{k}}^{(\lambda)}(\lambda=1,2)$. It is convenient then to discuss the electric field along a non-specified polarization $\vec{e}(\vec{k})$.

The transverse electric field operator in the non-relativistic QED is given by

$$
\vec{E}_{T}(\vec{x}, t)=-\frac{1}{c} \frac{\partial}{\partial t} \vec{A}_{T}(\vec{x}, t)
$$

with the transverse vector potential operator (radiation field) in the Heisenberg image of the free Hamiltonian being

$$
\vec{A}_{T}(\vec{x}, t)=\sqrt{\frac{\hbar c}{\Omega}} \sum_{\lambda=1,2} \sum_{\vec{q}} \frac{1}{\sqrt{|\vec{q}|}} \vec{e}_{\vec{q}}^{(\lambda)} e^{-\imath \vec{q} \vec{x}}\left(e^{-\imath c q t} b_{\vec{q}, \lambda}+e^{\imath c q t} b_{-\vec{q}, \lambda}^{+}\right)
$$

(with periodical boundary conditions) and

$$
\left[b_{\vec{q}, \lambda}, b_{\vec{q}^{\prime}, \lambda^{\prime}}^{+}\right]=\delta_{\vec{q}, \vec{q}^{\prime}} \delta_{\lambda, \lambda^{\prime}}
$$


We consider analogously to the treatment of the noise of the longitudinal electric field the two hermitian (scalar) operators constructed from the Fourier transform of the electrical field in the space variable

$$
\begin{aligned}
& E_{T}^{R}(\vec{k}, t)=\frac{1}{2} \vec{e}_{\vec{k}}\left(\overrightarrow{\tilde{E}}_{T}(\vec{k}, t)+\overrightarrow{\tilde{E}}_{T}(-\vec{k}, t)\right) \\
& E_{T}^{I}(\vec{k}, t)=-\imath \frac{1}{2} \vec{e}_{\vec{k}}\left(\overrightarrow{\tilde{E}}_{T}(\vec{k}, t)-\overrightarrow{\tilde{E}}_{T}(-\vec{k}, t)\right)
\end{aligned}
$$

for an arbitrary polarization direction $\vec{e}_{\vec{k}}$.

The noise of the observable $E_{T}^{R}$ according to Eq.8 is

$$
\tilde{\delta}_{E_{T}^{R}}(\omega, k)=\hbar \omega \operatorname{coth}\left(\frac{\beta \hbar \omega}{2}\right) \Re \int_{0}^{\infty} d t e^{-\imath \omega t} \int_{0}^{\beta} d \lambda\left\langle E_{T}^{R}(\vec{k}, 0) E_{T}^{R}(\vec{k}, t+\imath \hbar \lambda)\right\rangle_{0} .
$$

and analogously for $E_{T}^{I}$.

From this step on we shall neglect the retardation. Then since only the internal electric field fluctuates one may express the transverse electric field directly by the transverse current density

$$
E_{T}(\vec{k}, t)=-\frac{4 \pi}{(c k)^{2}} \frac{\partial}{\partial t} j_{T}(\vec{k}, t)
$$

where of course $j_{T}(\vec{k}, t)$ is the projection of the current density on the same chosen polarization direction. Consequently we consider the time evolution also in the $1 / c^{2}$ approximation i.e. in the frame of the Hamiltonian Eq. 13.

Thereafter we get, as in the longitudinal case the only surviving contribution

$$
\tilde{\delta}_{E_{T}}(\omega, k)=\frac{(4 \pi)^{2}}{(c k)^{4}} \hbar \omega \operatorname{coth}\left(\frac{\beta \hbar \omega}{2}\right) \Re \int_{0}^{\infty} d t e^{-\imath \omega t} \int_{0}^{\beta} d \lambda\left\langle\dot{j}_{T}(\vec{k}, 0) \dot{j}_{T}(-\vec{k}, t+\imath \hbar \lambda)\right\rangle_{0} .
$$

(Since this equation is valid for both fields $E_{T}^{R}$ and $E_{T}^{I}$ we omited the $R, I$ indices.) However, here the argument for the absence of the other contributions is due only to the presence of the Kronecker-delta $\delta_{\vec{k}}$ in front of them. For our final result relating the noise spectrum to the transverse conductivity these are anyway irrelevant since unlike in the longitudinal case, the wave vector and the frequency of the transverse field are not independent

Using also the invariance against space inversion it may be shown, that the real part of the integral over $\lambda$ is even in time, while the imaginary part is odd. Therefore, the time integration my be extended to $-\infty$, where the correlations are supposed to vanish.

Now, due to time translation invariance of the equilibrium average one may transfer the time derivation of the first current on the second one (implying a change of sign) and after two integration by parts one has

$$
\begin{gathered}
\int_{0}^{\infty} d t e^{-\imath \omega t}\left\langle\int_{0}^{\beta} d \lambda \dot{j}_{T}(\vec{k}, 0) \dot{j}_{T}(-\vec{k}, t+\imath \hbar \lambda)\right\rangle_{0}= \\
\omega^{2} \int_{0}^{\infty} d t e^{-\imath \omega t} \int_{0}^{\beta} d \lambda\left\langle j_{T}(\vec{k}, 0) j_{T}(-\vec{k}, t+\imath \hbar \lambda)\right\rangle_{0} .
\end{gathered}
$$


Further,

$$
\left\langle j_{T}(\vec{k}, 0) j_{T}(-\vec{k}, t+\imath \hbar \lambda)\right\rangle_{0}=\Omega \int d \vec{x} e^{i \vec{k} \vec{x}}\left\langle j_{T}(0,0) j_{T}(\vec{x}, t+\imath \hbar \lambda)\right\rangle_{0}
$$

and therefore we have finally

$$
\begin{aligned}
\tilde{\delta}_{E_{T}}(\omega, k) & =\Omega \omega^{2} \frac{(4 \pi)^{2}}{(c k)^{4}} \hbar \omega \operatorname{coth}\left(\frac{\beta \hbar \omega}{2}\right) \\
& \times \Re \int_{0}^{\infty} d t e^{-\imath \omega t} \int_{0}^{\beta} d \lambda \int d \vec{x} e^{\imath \vec{k} \vec{x}}\left\langle j_{T}(0,0) j_{T}(\vec{x}, t+\imath \hbar \lambda)\right\rangle_{0} .
\end{aligned}
$$

Now, from Eq.17 we had

$$
\kappa(k, \omega)_{T}=\int_{0}^{\infty} d t \int_{0}^{\beta} d \lambda \int d \vec{x} e^{\imath(\vec{k} \vec{x}+\omega t)}\left\langle j_{T}(0,0) j_{T}(\vec{x}, t+\imath \hbar \lambda)\right\rangle_{0}
$$

and using Eq.31

$$
\kappa(k, \omega)_{T}=\frac{\sigma_{T}(\omega, k)}{1-\imath \omega \frac{4 \pi}{c^{2} k^{2}} \sigma_{T}(k, \omega)} .
$$

At first glance it seems that we found a simple relationship between the noise of the transverse field and the transverse conductivity. However the correct interpretation is more subtle. In the case of the transverse conductivity the variables $\omega$ and $k$ are not independent: $\omega=c k$. Therefore, according to Eq. 32 one has just a frequency dependent conductivity and

$$
\kappa(\omega, \omega / c)_{T}=\frac{\sigma_{T}(\omega)}{1-\frac{4 \pi i}{\omega} \sigma_{T}(\omega)} .
$$

In the transverse noise spectral density $k$ is the wave vector of the electric field while $\omega$ is the frequency of the noise. They are independent variables. A direct relationship to the transverse conductivity however exists only along the line $\omega=c k$.

Thus

$$
\begin{aligned}
\left.\tilde{\delta}_{E_{T}}(\omega, k)\right|_{k=\omega / c} & =\Omega 4 \pi \hbar \frac{4 \pi}{\omega} \operatorname{coth}\left(\frac{\beta \hbar \omega}{2}\right) \Re \frac{\sigma_{T}(\omega)}{1-\frac{4 \pi \imath}{\omega} \sigma_{T}(\omega)} \\
& =\Omega 4 \pi \hbar \operatorname{coth}\left(\frac{\beta \hbar \omega}{2}\right) \Im \frac{1}{1-\frac{4 \pi \imath}{\omega} \sigma_{T}(\omega)}
\end{aligned}
$$

This is again a new important relationship.

\section{The noise spectrum of the photon occupation numbers.}

Beside the field noises one might consider also the noise of the photon occupation numbers. The noise spectral density of the photon occupation numbers $n_{\vec{k}}$ (with an arbitrary chosen polarization not mentioned) is given by 


$$
\tilde{\delta}_{n_{\vec{k}}}(\omega)=2 \hbar \omega \operatorname{coth}\left(\frac{\beta \hbar \omega}{2}\right) \Re \int_{0}^{\infty} d t e^{-\imath \omega t} \int_{0}^{\beta} d \lambda\left\langle\hat{n}_{\vec{k}} \hat{n}_{\vec{k}}(t+\imath \hbar \lambda\rangle_{0}\right.
$$

where $\hat{n}_{\vec{k}} \equiv b_{\vec{k}}^{+} b_{\vec{k}}$ is the operator of the number of photons of wave vector $\vec{k}$ and the given polarization we do not mention in the notation.

On the other hand analogously to Eq.73 we have

$$
\int_{0}^{\infty} d t e^{-\imath \omega t}\left\langle\hat{n}_{\vec{k}}(0) \hat{n}_{\vec{k}}(t+\imath \hbar \lambda)\right\rangle_{0}=\frac{1}{\omega^{2}} \int_{0}^{\infty} d t e^{-\imath \omega t}\left\langle\dot{\hat{n}}_{\vec{k}}(0) \dot{\hat{n}}_{\vec{k}}(t+\imath \hbar \lambda)\right\rangle_{0} .
$$

However, retaining the lowest order in $\frac{1}{c}$ from the full QED Hamiltonian

we have

$$
\frac{1}{c} \vec{j}_{T}(\vec{x}, t) \vec{A}_{T}
$$

and

$$
\dot{n}_{\vec{k}}(t)=\frac{\imath}{\hbar c} \int d \vec{x}\left[\vec{j}_{T}(\vec{x}, t) \vec{A}_{T}(\vec{x}, t), n_{\vec{k}}(t)\right]
$$

$$
\begin{aligned}
& \int_{0}^{\infty} d t e^{-\imath \omega t}\left\langle n_{\vec{k}}(0) n_{\vec{k}}(t+\imath \hbar \lambda)\right\rangle_{0}=-\frac{1}{(\hbar \omega c)^{2}} \int_{0}^{\infty} d t e^{-\imath \omega t} \int d \vec{x} \int d \vec{x}^{\prime} \\
& \left\langle\left[\vec{j}_{T}(\vec{x}, 0) \vec{A}_{T}(\vec{x}, 0), n_{\vec{k}}(0)\right]\left[\vec{j}_{T}\left(\vec{x}^{\prime}, t+\imath \hbar \lambda\right) \vec{A}_{T}\left(\vec{x}^{\prime}, t+\imath \hbar \lambda\right), n_{\vec{k}}(t+\imath \hbar \lambda)\right]\right\rangle_{0}
\end{aligned}
$$

where the transverse vector potential operator is defined by Eq. 66 .

In what follows we neglect as before retardation and ignore consequently terms higher order than $1 / c^{2}$. Within this approximation we remain only with an approximate Hamiltonian being the sum of the $\frac{1}{c^{2}}$ e.m Hamiltonian Eq. 13 (as well as some other interactions) for electrons and of the free Hamiltonian of the photons both in the averaging over equilibrium as in the time evolution.

Performing the commutations and taking again into account the translation, rotation and reflection invariance in the coordinate space we get after some algebra

$$
\begin{aligned}
& \tilde{\delta}_{n_{\vec{k}}}(\omega)=2 \frac{\operatorname{coth}\left(\frac{\beta \hbar \omega}{2}\right)}{c k \omega} \Re \int_{0}^{\infty} d t e^{-\imath \omega t} \int_{0}^{\beta} d \lambda\left\langle\tilde{j}(\vec{k}, 0) j_{T}(0, t+\imath \hbar \lambda)\right\rangle_{0}^{e} \times \\
& {\left[e^{-\imath(\omega-c k) t-c k \lambda}\left(1+\mathcal{N}_{k}\right)+e^{-\imath(\omega+c k) t+c k \lambda)} \mathcal{N}_{k}\right]}
\end{aligned}
$$

where

$$
\mathcal{N}_{k} \equiv \frac{1}{e^{\beta k c}-1}
$$

is the Bose distribution of photons.

The above expression resembles Eq. 75 for the noise of the transverse electric field, however they differ essentially due to the presence of the photonic factors alongside the electronic the current-current correlator under the integrals. Therefore, it cannot be related to the transverse conductivity. Nevertheless, Eq. 86 may be the starting point for the direct computation of the photon number noise spectrum within some approximations for homogeneous, isotropic solid state models. 


\section{Conclusions.}

We started from the generally accepted quantum statistical definition [12] of the thermal fluctuation of an observable as its average square deviation in time and the noise as its Fourier transform. We have chosen as relevant electromagnetic observables the real and imaginary parts of the Fourier transforms of the longitudinal $\tilde{E}_{L}(t, \vec{k})$ and transverse $\tilde{E}_{T}(t, \vec{k})$ electric fields as well as the photon number $n_{\vec{k}}(t)$ in a homogeneous isotropic system. These three kind of noise spectra we discussed exhaust the different experimental configurations of electromagnetic thermal fluctuations.

We use throughout the improved linear response theory according to the proper definition of the total field in a system of electromagnetically interacting charged particles according to [14] and [15] (see also Ref. [8]) to relate the thermal noise spectrum of the Fourier transform in the space variable of the "real" and "imaginary" (hermitian) parts of the longitudinal and transverse electric field operators to the frequency and wave-vector dependent complex dielectric function (or conductivity). In the case of a longitudinal field in the zero wave vector and zero frequency limit the classical Nyquist theorem [1] results, while for non-vanishing frequencies a modification of the result of Ref. [3] as it was predicted earlier in Ref.[4] emerges. Actually we got as well in the longitudinal as in the transverse case more general results including $k \neq 0$. However, in the transverse case the relationship to the transverse conductivity occurs obviously only for $\omega=c k$ and it was obtained from the QED within the $1 / c^{2}$ approximation i.e. after neglecting retardation.

Finally, we discussed also the noise spectrum of the photon occupation numbers within the same $1 / c^{2}$ approximation. This noise spectrum is again related to the transverse electronic current-current correlator. However, due to the presence of photonic factors under the integrals it cannot be related to the transverse conductivity.

It is important to mention, that in our derivations no other ingredients were introduced except the implicit assumption of linear response theory about the time-decay of correlations (i.e. irreversibility in the thermodynamic limit).

\section{References}

[1] H. Nyquist, Phys. Rev. 31,110 (1928)

[2] J.B. Johnson, Phys. Rev. 32, 97 (1928)

[3] H.B. Callen, T.A. Welton, Phys. Rev. 8334 (1951)

[4] L.Bányai, A. Aldea and P. Gartner, Z. Phys. B 58,161 (1985)

[5] V.I. Tatarski, Usp. Fiz. Nauk., 151, 273 (1987)

[6] Yu. L. Klimontovich, Usp. Fiz. Nauk., 151, 309 (1987)

[7] V.L. Ginzburg and L.O. Pitaevskii, Usp. Fiz. Nauk., 151, 333 (1987) 
[8] Ladislaus Alexander Bányai, Lectures on Non-Equilibrium Theory of Condensed Matter. Second Edition. World Scientific (2020)

[9] U. Marini, B. Marconi, A. Puglisi, L. Rondoni and A. Vulpiani, Phys. Rep. 461, Issues 4-6, pp. 111- 195 (2008)

[10] A. A. Clerk, M. H. Devoret, S. M. Girvin, F. Marquardt, and R. J. Schoelkopf, Rev. Mod. Phys. 82, 1155 (2010)

[11] L. Reggiani and E. Alfinito,Fluctuation and Noise Letters 18, NO. 01 DOI 10.1142/S0219477519300015 (2018)

[12] E.M. Lifshitz, L.P. Pitaevskii, Statistical Phyics. Part 2. Oxford: Pergamon Press (1980)

[13] R. Kubo, J. Phys. Soc. Jpn, bf 12, 570 (1957)

[14] T. Izuyama, Progr. Theor. Phys. bf 25,964 (1961)

[15] D. N. Zubarev, Nonequilibrium statistical thermodynamics. New York, London, Consultants Bureau 1974

[16] N. Wiener, Norbert (1930 Acta Mathematica. 55, 117 (1930)

[17] A.Khintchine, Mathematische Annalen. 109, (1): 604 (1930)

[18] S.R. de Groot and P. Mazur, Non-Equilibrium Thermodynamics, Amsterdam, North-Holland Publ. Co. (1962)

[19] C. G. Darwin, Phil. Mag. ser. 6. 36, 537 (1920)

[20] L.A. Bányai, Int. J. Theor. Phys. (2021) DOI 10.1007/s10773-021-04842-9

[21] O.V. Dolgov, D.A. Kirzhnits, E.G. Maksimov, Rev. Mod. Phys, 53, 81 (1981) 